Original Research Paper

\title{
Fitted Reproducing Kernel Method for Solving a Class of Third-Order Periodic Boundary Value Problems
}

\author{
${ }^{1}$ Asad Freihat, ${ }^{2}$ Radwan Abu-Gdairi, ${ }^{3}$ Hammad Khalil, \\ ${ }^{4}$ Eman Abuteen, ${ }^{1}$ Mohammed Al-Smadi and ${ }^{3}$ Rahmat Ali Khan \\ ${ }^{1}$ Department of Applied Science, Ajloun College, Al Balqa Applied University, Ajloun 26816, Jordan \\ ${ }^{2}$ Department of Mathematics, Faculty of Science, Zarqa University, Zarqa 13110, Jordan \\ ${ }^{3}$ Department of Mathematics, University of Malakand, Chakadara Dir(L), Khyber Pakhtunkhwa, Pakistan \\ ${ }^{4}$ Department of Applied Science, Faculty of Engineering Technology, Al-Balqa Applied University, Amman 11942, Jordan
}

Article history

Received: 09-12-2015

Revised: 27-04-2016

Accepted: 02-05-2016

Corresponding Author:

Mohammed Al-Smadi

Department of Applied

Science, Ajloun College, Al-

Balqa Applied University,

Ajloun 26816, Jordan

Email:mhm.smadi@yahoo.com

\begin{abstract}
In this article, the reproducing kernel Hilbert space $W_{2}^{4}[0,1]$ is employed for solving a class of third-order periodic boundary value problem by using fitted reproducing kernel algorithm. The reproducing kernel function is built to get fast accurately and efficiently series solutions with easily computable coefficients throughout evolution the algorithm under constraint periodic conditions within required grid points. The analytic solution is formulated in a finite series form whilst the truncated series solution is given to converge uniformly to analytic solution. The reproducing kernel procedure is based upon generating orthonormal basis system over a compact dense interval in sobolev space to construct a suitable analytical-numerical solution. Furthermore, experiments results of some numerical examples are presented to illustrate the good performance of the presented algorithm. The results indicate that the reproducing kernel procedure is powerful tool for solving other problems of ordinary and partial differential equations arising in physics, computer and engineering fields.
\end{abstract}

Keywords: Boundary Value Problem, Error Estimation and Error Bound, Reproducing Kernel Theory

\section{Introduction}

Periodic Boundary Value Problem (PBVP) is an active research of modern mathematics that can be found naturally in different branches of applied sciences, physics and engineering (Gregu, 1987; Minh, 1998; Ashyralyev et al., 2009). It has many applications due to the fact that a lot practical problems in mechanics, electromagnetic, astronomy and electrostatics may be converted directly to such PBVP. However, it is difficult generally to get a closed form solution for PBVP in terms of elementary functions, especially, for nonlinear and nonhomogeneous cases. So, PBVP has attracted much attention and has been studied by several authors (Kong and Wang, 2001; Chu and Zhou, 2006; Liu et al., 2007; Zehour et al., 2008; Yu and Pei, 2010; Abu Arqub and Al-Smadi, 2014). The purpose of this analysis is to develop analytical-numerical method for handling third-order, two-point PBVP with given periodic conditions by an application of the reproducing kernel theory. More specifically, consider the general form of third-order BVP: $y^{\prime \prime \prime}(t)=F\left(t, y(t), y^{\prime}(t), y^{\prime \prime}(t)\right), 0 \leq t \leq 1$

with periodic conditions:

$y(0)-y(1)=0, y^{\prime}(0)-y^{\prime}(1)=0, y^{\prime \prime}(0)-y^{\prime \prime}(1)=0$

where, $\mathrm{y} \in y \in W_{2}^{4}[0,1]$ is unknown function to be determined, $F\left(t, v_{1}, v_{2}, v_{3}\right)$ is continuous in $W_{2}^{1}[0,1]$ as $v_{i}=v_{i}(t) \in W_{2}^{4}[0,1], 0 \leq t \leq 1,-\infty<v_{i}<1, i=1,2,3$, which is linear or nonlinear term depending on the problem discussed.

The numerical solvability of BVPs with periodic conditions of different order has been pursued in literature. To mention a few, the existence and multiplicity of positive solutions for PBVP in the forms $u^{\prime \prime \prime}(x)=\alpha(x) f(x, u(x)), u^{\prime \prime \prime}$ $(x)=f(x, u(x))+\rho u(x)$ and $\mathrm{u}^{\prime \prime \prime}(x)=\rho u^{\prime \prime}(x)+f(x, u(x))+$ $\alpha(x)$ have been studied, respectively, by Liu et al. (2007), Chu and Zhou (2006) and Yu and Pei (2010). However, Al-Smadi et al. (2014) have developed an iterative method 
for handling system of first-order PBVPs based on the RKHM. While Hopkins and Kosmatov (2007) have provided the existence of at least one positive solutions of PBVP in the form $u^{\prime \prime \prime}(x)=f\left(x, u(x), u^{\prime}(x), u^{\prime \prime}(x)\right)$. Further, Taylors decomposition method for solving linear periodic equation $u^{\prime \prime \prime}(x)=\alpha_{1}(x) u^{\prime \prime}(x)+\alpha_{2}(x) u^{\prime}(x)+\alpha_{3}(x) u(x)+$ $\alpha_{4}(x)$ numerically is proposed by Ashyralyev et al. (2009). The reproducing kernel method has widely applications to construct a numeric-analytic solution of different types of IVPs and BVPs. For example, see the work in (Geng and Cui, 2012; Al-Smadi et al., 2012; 2013; Komashynska and Al-Smadi, 2014; Abu-Gdairi and Al-Smadi, 2013; Abu Arqub et al., 2012; 2013; 2015; Geng and Qian, 2013; Bushnaq et al., 2016; Ahmad et al., 2016) for more information's about RKHS method and scientific applications. But on the other aspect as well, several numerical schemes have been applied to solve IVPs and BVPs. For example, we refer to the work in (Komashynska et al., 2016a; 2016b; 2016c; Momani et al., 2014; Abu-Gdairi et al., 2015; Al-Smadi et al., 2015; 2016; Al-Smadi, 2013; Moaddy et al., 2015; El-Ajou et al., 2015; Abuteen et al., 2016).

The structure of this article is organized as follows. In section 2, reproducing kernel spaces are described to compute its reproducing kernels functions in which every function satisfies the periodic conditions. In section 3 and 4, the analytical-numerical solutions of Equation 1 and 2 as well an iterative method for obtaining these solutions are presented with series formula in the space $W_{2}^{4}[0,1]$. The n-term numerical solution is obtained to converge uniformly to analytic solution. In section 5, some numerical examples are simulated to check the reasonableness of our theory and to demonstrate the high performance of the presented algorithm. Conclusions are summarized in the last section.

\section{Construction of Reproducing Kernel Functions}

In this section, we present some basic results and remarks in the reproducing kernel theory and its applications.

\section{Definition 1}

Let $W_{2}^{4}[0,1]=\left\{y \mid y, y^{\prime}, y^{\prime \prime}, y^{\prime \prime \prime}\right.$ are absolutely continuous on $[0,1]$ such that $y^{(4)} \in L^{2}[0,1]$ and $y(0)-y$ $\left.(1)=0, y^{\prime}(0)-y^{\prime}(1)=0, y^{\prime \prime}(0)-y^{\prime \prime}(1)=0\right\}$. On the other hand, let $\langle y(t), z(t)\rangle_{W_{2}^{4}}$ be the inner product in the space $W_{2}^{4}[0,1]$, which is defined by:

$$
\langle y(t), z(t)\rangle W_{2}^{4}=\sum_{i=0}^{3} y^{(i)}(0) z^{(i)}(0)+\int_{0}^{1} y^{(4)}(v) z^{(4)}(v) d v
$$

and the norm is $\|y\|_{W_{2}^{4}}=\sqrt{\langle y(t), y(t)\rangle W_{2}^{4}}$, where $y, z \in W_{2}^{4}[0,1]$.

\section{Lemma 1}

The reproducing kernel function $K_{s}(t)$ of the Hilbert space $W_{2}^{4}[0,1]$ can be given by:

$$
K_{s}(t)= \begin{cases}\sum_{i=0}^{7} a_{i}(s) t^{i}, & t \leq s \\ \sum_{i=0}^{7} b_{i}(s) t^{i}, & t>s\end{cases}
$$

\section{Proof}

Using several integration by parts of $\int_{0}^{1} y^{(4)}(v) K_{s}^{(4)}(v) d v$ to obtain that:

$$
\begin{aligned}
& \left\langle y(t), K_{s}(t)\right\rangle_{W_{2}^{4}}=\sum_{i=0}^{3} y^{(i)}(0)\left[\partial_{t}^{i} K_{s}(0)+(-1)^{i} \partial_{t}^{7-i} K_{s}(0)\right] \\
& +\sum_{i=0}^{3}(-1)^{i+1} y^{(i)}(1) \partial_{t}^{7-i} K_{s}(1)+\int_{0}^{1} y(v) \partial_{t}^{8} K_{s}(v) d v
\end{aligned}
$$

If $K_{s}(t) \in W_{2}^{4}[0,1]$, then $K_{s}(0)=K_{s}(1), \partial_{t} K_{s}(0)=$ $\partial_{t} K_{s}(1), \partial_{t}^{2} K_{s}(0)=\partial_{t}^{2} K_{s}(1)$, as well as if $y \in W_{2}^{4}[0,1]$, then $y^{(i)}(0)=y^{(i)}(1), i=0,1,2$. Therefore:

$$
\begin{aligned}
& \left\langle y(t), K_{s}(t)\right\rangle_{W_{2}^{4}}=\sum_{i=0}^{3} y^{(i)}(0)\left[\partial_{t}^{i} K_{s}(0)+(-1)^{i} \partial_{t}^{7-i} K_{s}(0)\right] \\
& +\sum_{i=0}^{3}(-1)^{i+1} y^{(i)}(1) \partial_{t}^{7-i} K_{s}(1)+\int_{0}^{1} y(v) \partial_{t}^{8} K_{s}(v) d v \\
& +c_{1}(y(0)-y(1))+c_{2}\left(y^{\prime}(0)-y^{\prime}(1)\right)+c_{3}\left(y^{\prime \prime}(0)-y^{\prime \prime}(1)\right)
\end{aligned}
$$

For each $s, t \in[0,1]$, assume $K_{s}(t)$ satisfy the following:

$$
\begin{aligned}
& \partial_{t}^{4} K_{s}(1)=0, \partial_{t}^{3} K_{s}(0)-\partial_{t}^{4} K_{s}(0)=0, \\
& K_{s}(0)+\partial_{t}^{7} K_{s}(0)+c_{1}=0, \\
& \partial_{t}^{7} K_{s}(1)+c_{1}=0, \partial_{t}^{6} K_{s}(1)-c_{2}=0, \\
& \partial_{t} K_{s}(0)-\partial_{t}^{6} K_{s}(0)+c_{2}=0, \\
& \partial_{t}^{2} K_{s}(0)+\partial_{t}^{5} K_{s}(0)+c_{3}=0, \\
& \partial_{t}^{5} K_{s}(1)+c_{3}=0
\end{aligned}
$$

Thus, we have $\left\langle y(t), K_{s}(t)\right\rangle_{W_{2}^{4}}=\int_{0}^{1} y(v) \partial_{t}^{8} K_{s}(v) d v$. Also, assume $K_{s}(t)$ satisfy that:

$\partial_{t}^{8} K_{s}(t)=\delta(s-t), \delta$ dirac - delta function

so, $\left\langle y(t), K_{s}(t)\right\rangle_{W_{2}^{4}}=y(s)$. 
Next, we give the expression form of $K_{s}(t)$, to do this, the auxiliary formula of Equation 5 is $\lambda^{8}=0$ and their real solutions are $\lambda=0$ with multiplicity 8 . Hence, let the form of $K_{s}(t)$ be as defined in Equation 4. On the other hand of Equation 5, let $K_{s} \quad(t)$ satisfy $\partial_{t}^{8} K_{s}(t+0)=\partial_{t}^{m} K_{s}(t-0), m=0,1, \ldots, 6$. By integrating $\partial_{t}^{8} K_{s}(t)=\delta(s-t)$ from $s-\varepsilon$ to $s+\varepsilon$ with respect to $t$ as well letting $\varepsilon \rightarrow 0$, we have the jump degree of $\partial_{t}^{7} K_{s}(t)$ at $s=t$ such that $\partial_{t}^{7} K_{s}(t+0)-\partial_{t}^{7} K_{s}(t-0)=-1$.

Through the last computational results the unknown coefficients $a_{i}(s)$ and $b_{i}(s), i=0,1, \ldots, 7$ of $K_{s}(t)$ in Equation 4 can be obtained. However, the representation form of these coefficients using Maple 13 software package are provided by:

$$
\begin{aligned}
& a_{0}(s)=1 \text {, } \\
& a_{1}(s)=\frac{1}{\alpha_{1}} s\left(9244-6300 s-50820 s^{2}-12705 s^{3}+205611 s^{4}-203035 s^{5}+58005 s^{6}\right), \\
& a_{2}(s)=\frac{1}{\alpha_{2}} s\left(-25200+2041264 s-3024100 s^{2}-756025 s^{3}+2570499 s^{4}-806113 s^{5}+5 s^{6}\right), \\
& a_{3}(s)=\frac{1}{\alpha_{3}} s\left(-1829520-27216900 s+72804784 s^{2}, 54887415 s^{3}+11205561 s^{4}-76873 s^{5}+363 s^{6}\right), \\
& a_{5}(s)=\frac{1}{\alpha_{4}} s\left(-1829520-27216900 s+72804784 s^{2}, 54887415 s^{3}+11205561 s^{4}-76873 s^{5}+363 s^{6}\right) \text {, } \\
& a_{5}(s)=\frac{1}{\alpha_{5}} s\left(49346640-138124504 s+74703740 s^{2}+18675935 s^{3}-5054705 s^{4}+462685 s^{5}-9791 s^{6}\right) \text {, } \\
& a_{6}(s)=\frac{1}{\alpha_{6}} s\left(146169244-145159740 s-1537460 s^{2}-384365 s^{3}+1388055 s^{4}-504739 s^{5}+29005 s^{6}\right), \\
& a_{7}(s)=\frac{1}{\alpha_{7}}\left(-292354444+292345200 s+6300 s^{2}+50820 s^{3}+12705 s^{4}-205611 s^{5}+203035 s^{6}-58005 s^{7}\right) \text {, } \\
& b_{0}(s)=1-\frac{1}{5040} s^{7}, \\
& b_{1}(s)=\frac{1}{\alpha_{1}} s\left(9244-6300 s-50820 s^{2}-12705 s^{3}+205611 s^{4}+\frac{36542311}{180} s^{5}+58005 s^{6}\right) \text {, } \\
& b_{2}(s)=\frac{1}{\alpha_{2}} s\left(-25200+2041264 s-3024100 s^{2}-756025 s^{3}-\frac{34351126}{15} s^{4}-806443 s^{5}+5 s^{6}\right) \text {, } \\
& b_{3}(s)=\frac{1}{\alpha_{3}} s\left(-1829520-27216900 s+72804784 s^{2}+18201196 s^{3}+11205561 s^{4}-76873 s^{5}+363 s^{6}\right) \text {, } \\
& b_{4}(s)=\frac{1}{\alpha_{4}} s\left(-1829520-27216900 s+219549660 s^{2}-54887415 s^{3}+11205561 s^{4}-76873 s^{5}+363 s^{6}\right) \text {, } \\
& b_{5}(s)=\frac{1}{\alpha_{5}} s\left(49346640+154229940 s+74703740 s^{2}+18675935 s^{3}-5054705 s^{4}+462685 s^{5}, 9791 s^{6}\right) \text {, } \\
& b_{6}(s)=\frac{1}{\alpha_{6}} s\left(-146185200-145159740 s-1537460 s^{2}-384365 s^{3}+1388055 s^{4}-504739 s^{5}+29005 s^{6}\right) \text {, } \\
& b_{7}(s)=\frac{1}{\alpha_{7}} s\left(292345200+6300 s+50820 s^{2}+12705 s^{3}-205611 s^{4}+203035 s^{5}-58005 s^{6}\right)
\end{aligned}
$$

where, $\alpha_{1}=292354444, \alpha_{2}=1169417776, \alpha_{3}=$ $10524759984, \alpha_{4}=42099039936, \alpha_{5}=70165066560$, $\alpha_{6}=210495199680$ and $\alpha_{7}=1473466397760$.

\section{Definition 2}

Let $W_{2}^{1}[0,1]=\{y \mid y$ is absolutely continuous on $[0,1]$ and $\left.y^{\prime} \in L^{2}[0,1]\right\}$ and let the inner product $\langle y(t), z(t)\rangle_{W_{2}^{1}}$ be written as:

$$
\langle y(t), z(t)\rangle_{w_{2}^{1}}=y(0) z(0)+\int_{0}^{1} y^{\prime}(v) z^{\prime}(v) d v
$$

whereas the norm \|\|$_{W_{2}^{\prime}}$ is given such that $\|y\|_{W_{2}^{1}} \sqrt{\langle y(t), y(t)\rangle_{W_{2}^{1}}}, y, z \in W_{2}^{1}[0,1]$.

However, Geng and Cui (2007) show that the reproducing kernel function $Q_{s}(t)$ of $W_{2}^{1}[0,1]$ can be given by: 


$$
Q_{s}(t)= \begin{cases}1+t & t \leq s \\ 1+s & t>s\end{cases}
$$

\section{Representation of Analytical-Numerical Solutions}

First, as in (Geng et al., 2013; 2014; 2015; Al-Smadi and Altawallbeh, 2013; Abu Arqub, 2015), we transform the problem into a differential operator. To do so, we define an operator $T$ from the space $W_{2}^{4}[0,1]$ into $W_{2}^{1}[0,1]$ such that $T y(t)=y^{\prime \prime \prime}(t)$. Therefore, Equation 1 and 2 can be converted equivalently into following form:

$$
T y(t)=F\left(t, y(t), y^{\prime}(t), y^{\prime \prime}(t)\right), 0 \leq t \leq 1
$$

with periodic conditions:

$$
y(0)-y(1)=0, y^{\prime}(0)-y^{\prime}(1)=0, y^{\prime \prime}(0)-y^{\prime \prime}(1)=0
$$

where, $y \in W_{2}^{4}[0,1]$ and $F\left(t, v_{1}, v_{2}, v_{3}\right) \in W_{2}^{1}[0,1]$ for $v_{i}=$ $v_{i}(t) \in W_{2}^{4}[0,1], \infty<v_{i}<\infty, i=1,2,3$ and $0 \leq t \leq 1$.

\section{Lemma 2}

The operator $T$ is linear bounded operator from $W_{2}^{4}[0,1]$ into $W_{2}^{1}[0,1]$.

\section{Proof}

We want to show that $\|T y\|_{W_{2}^{1}}^{2} \leq M\|y\|_{W_{2}^{4}}^{2}$, where $M>0$. From Definition 2, we get that:

$$
\begin{gathered}
\|T y\|_{W_{2}^{1}}^{2}=\langle T y(t), T y(t)\rangle_{W_{2}^{1}}=[(T y)(0)]^{2}+\int_{0}^{1}\left[(T y)^{\prime}(t)\right]^{2} d t \\
\text { Since } y(s)=\left\langle y(t), K_{s}(t)\right\rangle_{W_{2}^{4}}, \quad(T y)(s)= \\
\left\langle y(t),\left(T K_{s}\right)(t)\right\rangle_{W_{2}^{4}} \text { and }(T y)^{\prime}(s)=\left\langle y(t),\left(T K_{s}\right)^{\prime}(t)\right\rangle_{W_{2}^{4}} \text {, we }
\end{gathered}
$$
have by using Schwarz's inequality that:

$$
|(T y)(s)|=\left|\left\langle y(s),\left(T K_{s}\right)(s)\right\rangle_{W_{2}^{4}}\right| \leq\left\|T K_{s}\right\|_{W_{2}^{4}}\|y\|_{W_{2}^{4}}=M_{1}\|y\|_{W_{2}^{4}}
$$

where $M_{1}>0$. Also, $\left|(T y)^{\prime}(s)\right|=\left|\left\langle y(s),\left(T K_{s}\right)^{\prime}(s)\right\rangle_{w_{2}^{4}}\right| \leq$ $\left\|\left(T K_{s}\right)^{\prime}\right\|_{W_{2}^{4}}\|y\|_{W_{2}^{4}}=M_{1}\|y\|_{W_{2}^{4}}$, where $M_{2}>0$. Thus, $\|T y\|_{W_{2}^{1}}^{2}=[(T y)(0)]^{2}+\int_{0}^{1}\left[(T y)^{\prime}(t)\right]^{2} d t \leq\left(M_{1}^{2}+M_{2}^{2}\right)\|y\|_{W_{2}^{4}}^{2}$. The linearity part is obvious.
Now, to construct an orthonormal basis system $\left\{\bar{\psi}_{i}(t)\right\}_{i=1}^{\infty}$ of the space $W_{2}^{4}[0,1]$, we set firstly $\varphi_{i}(t)=$ $K_{t_{i}}(t)$ and $\psi_{i}(t)=T_{i}^{*} \varphi(t)$, where $\left\{t_{i}\right\}_{i=1}^{\infty}$ is dense set on compact $[0,1]$ and $T^{*}$ is the adjoint of $T$. Thus, we have that $\left\langle y(t), \psi_{i}(t)\right\rangle_{W_{2}^{4}}=\left\langle y(t), T^{*} \varphi_{i}(t)\right\rangle_{W_{2}^{4}}=\left\langle T y(t), \varphi_{i}(t)\right\rangle_{W_{2}^{1}}$ $=T y\left(t_{i}\right) i=1,2, \ldots$.

\section{Theorem 1}

Let $\left\{t_{i}\right\}_{i=1}^{\infty}$ be a dense subset on $[0,1]$, then $\left\{\psi_{i}(t)\right\}_{i=1}^{\infty}$ is complete basis system of $W_{2}^{4}[0,1]$.

\section{Proof}

It is easy to note that $\psi_{i}(t)=T^{*} \varphi_{i}(t)=$ $\left\langle T^{*} \varphi_{i}(t), K_{s}(t)\right\rangle_{w_{2}^{4}}=\left\langle\varphi_{i}(t), T_{t} K_{s}(t)\right\rangle_{w_{2}^{1}}=\left.T_{t} K_{s}(t)\right|_{y=x_{i}} \in$ $W_{2}^{4}[0,1]$. Therefore, $\psi_{i}(t)$ can be given by $\psi_{i}(t)=$ $\left.T_{t} K_{s}(t)\right|_{t=s_{s}}$, where $T_{t}$ refers to the operator $T$ applies to the function of $t$. However, let $\left\langle y(t), \psi_{i}(t)\right\rangle_{W_{2}^{4}}=0, i=1$, $2, \ldots$ for each fixed $t \in W_{2}^{4}[0,1]$. That is, $\left\langle y(t), \psi_{i}(t)\right\rangle_{W_{2}^{4}}=$ $\left\langle y(t), T^{*} \varphi_{i}(t)\right\rangle_{W_{2}^{4}}=\left\langle T y(t), \varphi_{i}(t)\right\rangle_{W_{2}^{1}}=T y\left(t_{i}\right)=0$. Since $\left\{t_{i}\right\}_{i=1}^{\infty}$ is dense on $[0,1]$, thus $T y(t)=0$ which means that $y(t)=0$.

Furthermore, $\left\{\bar{\psi}_{i}(t)\right\}_{i=1}^{\infty}$ can be obtained by using the Gram-Schmidt process of $\left\{\psi_{i}(t)\right\}_{i=1}^{\infty}$ as follows:

$$
\bar{\psi}_{i}(t)=\sum_{k=1}^{i} \beta_{i k} \psi_{k}(t)
$$

\section{Theorem 2}

Let $y(t)$ be analytic solution of Equation 7 and 8 and let $\left\{t_{i}\right\}_{i=1}^{\infty}$ be a dense subset on $[0,1]$, then:

$$
\begin{aligned}
& y(t)=T^{-1} F\left(t, y(t), y^{\prime}(t), y^{\prime \prime}(t)\right) \\
& =\sum_{i=1}^{\infty} \sum_{k=1}^{i} \beta_{i k} F\left(t_{k}, y\left(t_{k}\right), y^{\prime}\left(t_{k}\right), y^{\prime \prime}\left(t_{k}\right)\right) \bar{\psi}_{i}(t)
\end{aligned}
$$

\section{Proof}

Clearly that $\sum_{i=1}^{\infty}\left\langle y(t), \bar{\psi}_{i}(t)\right\rangle \bar{\psi}_{i}(t)$ is Fourier series expansion about orthonormal basis $\left\{\bar{\psi}_{i}(t)\right\}_{i=1}^{\infty}, y \in W_{2}^{4}[0$, 1]. Anyhow, since $W_{2}^{4}[0,1]$ is the Hilbert space, then 
$\sum_{i=1}^{\infty}\left\langle y(t), \bar{\psi}_{i}(t)\right\rangle \bar{\psi}_{i}(t)$ is convergent in the sense of $\|\cdot\|_{W_{2}^{4}}$.

Whilst by Equation 9, we have that:

$$
\begin{aligned}
& y(t)=\sum_{i=1}^{\infty}\left\langle y(t), \bar{\psi}_{i}(t)\right\rangle_{W_{2}^{4}} \bar{\psi}_{i}(t) \\
& =\sum_{i=1}^{\infty} \sum_{k=1}^{i} \beta_{i k}\left\langle y(t), \psi_{k}(t)\right\rangle_{W_{2}^{4}} \bar{\psi}_{i}(t) \\
& =\sum_{i=1}^{\infty} \sum_{k=1}^{i} \beta_{i k}\left\langle y(t), T^{*} \varphi_{k}(t)\right\rangle_{W_{2}^{4}} \bar{\psi}_{i}(t) \\
& =\sum_{i=1}^{\infty} \sum_{k=1}^{i} \beta_{i k}\left\langle T y(t), \varphi_{k}(t)\right\rangle_{W_{2}^{1}} \bar{\psi}_{i}(t) \\
& =\sum_{i=1}^{\infty} \sum_{k=1}^{i} \beta_{i k}\left\langle F\left(t, y(t), y^{\prime}(t), y^{\prime \prime}(t)\right), \varphi_{k}(t)\right\rangle_{W_{2}^{1}} \bar{\psi}_{i}(t) \\
& =\sum_{i=1}^{\infty} \sum_{k=1}^{i} \beta_{i k} F\left(t_{k}, y\left(t_{k}\right), y^{\prime}\left(t_{k}\right), y^{\prime \prime}\left(t_{k}\right)\right) \bar{\psi}_{i}(t) \\
& =T^{-1} F\left(t, y(t), y^{\prime}(t), y^{\prime \prime}(t)\right)
\end{aligned}
$$

Let $\left\{\bar{\psi}_{i}(t)\right\}_{i=1}^{\infty}$ be the orthonormal basis obtained by Gram-Schmidt process of $\left\{\psi_{i}(t)\right\}_{i=1}^{\infty}$, then the analytic solution of Equation 7 and 8 can be written as follows:

$$
y(t)=\sum_{i=1}^{\infty} B_{i} \bar{\psi}_{i}(t)
$$

where, $\sum_{k=1}^{i} \beta_{i k} F\left(t_{k}, y\left(t_{k}\right), y^{\prime}\left(t_{k}\right), y^{\prime \prime}\left(t_{k}\right)\right)$. Indeed, $B_{i}$ are unknown. Thus, we can approximate $B_{i}$ using known $A_{i}$. For computations, define the initial guess function $y_{0}\left(t_{1}\right)$ $=0$, set $y_{0}\left(t_{1}\right)=y\left(t_{1}\right)$ and define the $n$ th-order approximation $y_{n}(t)$ to $y(t)$ as follows:

$$
y_{n}(t)=\sum_{i=1}^{n} A_{i} \bar{\psi}_{i}(t)
$$

where, the coefficients $A_{i}$ of $\bar{\psi}_{i}(t), i=1,2, \ldots, n$ are obtained by:

$$
A_{i}=\sum_{k=1}^{i} \beta_{i k} F\left(t_{k}, y_{k-1}\left(t_{k}\right), y_{k-1}^{\prime}\left(t_{k}\right), y_{k-1}^{\prime \prime}\left(t_{k}\right)\right)
$$

\section{Theorem 3}

If $y \in W_{2}^{4}[0,1]$, then there exists $M>0$ such that $\left\|y^{(i)}\right\|_{C} \leq M\|y\|_{W_{2}^{4}}, i=0,1,2,3$, where $\|y\|_{C}=\max _{0 \leq t \leq 1}|y(t)|$.

\section{Proof}

For each $s, \quad t \in[0,1]$, we have $y^{(i)}(t)=$ $\left\langle y(t), \partial_{t}^{i} K_{s}(t)\right\rangle W_{2}^{4}, i=0,1,2,3$. By the expression form of $K_{s}(t)$, it follows that $\left\|\partial_{t}^{i} K_{s}\right\| W_{2}^{4} \leq M_{i}, i=0,1,2,3$, where $M_{i}>0$. Thus, $\quad\left|y^{(i)}(t)\right|=\left|\left\langle y(t), \partial_{t}^{i} K_{s}(t)\right\rangle_{W_{2}^{4}}\right| \leq$ $\left\|\partial_{t}^{i} K_{s}\right\|_{W_{2}^{4}}\|y\|_{W_{2}^{4}} \leq M_{i}\|y\|_{W_{2}^{4}}, i=0,1,2,3$. Hence, $\left\|y^{(i)}\right\|_{C} \leq$ $\max _{i=0,1,2,3}\left\{M_{i}\right\}\|y\|_{W_{2}^{4}}, i=0,1,2,3$.

\section{Corollary 1}

The numeric solution and its derivatives up to order three are converge uniformly to analytic solution and all its derivatives, respectively.

\section{Proof}

For each $t \in[0,1]$, we have:

$$
\begin{aligned}
& \left|y_{n}^{(i)}(t)-y^{(i)}(t)\right|=\left|\left\langle y_{n}(t)-y(t), \partial_{t}^{i} K_{s}(t)\right\rangle W_{2}^{4}\right| \\
& \leq\left\|\partial_{t}^{i} K_{x}\right\|_{W_{2}^{4}}\left\|y_{n}-y\right\| W_{2}^{4} \\
& \leq M_{i}\left\|y_{n}-y\right\|_{W_{2}^{4}}, i=0,1,2,3
\end{aligned}
$$

where, $M_{i}>0$. Therefore, if $\left\|y_{n}-y\right\|_{W_{2}^{4}} \rightarrow 0$ as $n \rightarrow 1$, then the numeric solution $y_{n}(x)$ and $y_{n}^{(i)}(t), i=1,2,3$, are converge uniformly to analytic solution $y(t)$ and $y^{(i)}(t), i$ $=1,2,3$, respectively.

\section{Convergence Analysis and Error Estimation}

From Corollary 1, if $y_{n}(t)$ converging uniformly to $y(t)=\sum_{i=1}^{\infty} A_{i} \bar{\psi}_{i}(t)$. If $y_{n}(t)=P_{n} y(t)$, where $P_{n}$ is orthogonal project from the space $W_{2}^{4}[0,1]$ to $\operatorname{Span}\left\{\psi_{1}\right.$, $\left.\psi_{2}, \ldots, \quad \psi_{n}\right\}, \quad$ then $T y_{n} \quad\left(t_{j}\right)=\left\langle T y_{n}(t), \varphi_{j}(t)\right\rangle_{W_{2}^{1}}=$ $\left\langle y_{n}(t), T_{j}^{*} \varphi(t)\right\rangle_{W_{2}^{4}}=\left\langle P_{n} y(t), \psi_{j}(t)\right\rangle_{W_{2}^{4}}=\left\langle y(t), P_{n} \psi_{j}(t)\right\rangle_{W_{2}^{4}}=$ $\left\langle y(t), \psi_{j}(t)\right\rangle_{w_{2}^{4}}=\left\langle T y(t), \varphi_{j}(t)\right\rangle_{w_{2}^{1}}=T y\left(t_{j}\right)$. Next, we list two lemmas for convenience in order to prove the recent theorems.

\section{Lemma 3}

The numerical solution $y_{n}$ satisfies, $T y_{n}\left(t_{j}\right)=$ $F\left(t_{j}, y_{j-1}\left(t_{j}\right), y_{j-1}^{\prime}\left(t_{j}\right), y_{j-1}^{\prime \prime}\left(t_{j}\right)\right)$ for $j=1,2,3$,

\section{Proof}

Let $A_{i}=\sum_{k=1}^{i} \beta_{i k} F\left(t_{k}, y_{k-1}\left(t_{k}\right), y_{k-1}^{\prime}\left(t_{k}\right), y_{k-1}^{\prime \prime}\left(t_{k}\right)\right)$, then $y_{n}$ will be rewritten in form of $y_{n}(t)=\sum_{i=1}^{n} A_{i} \psi_{i}(t)$. Using the properties of $K_{s}(t)$, it follows that: 


$$
\begin{aligned}
& T y_{n}\left(t_{j}\right)=\sum_{i=1}^{n} A_{i} T \psi_{i}\left(t_{j}\right)=\sum_{i=1}^{n} A_{i}\left\langle T \bar{\psi}_{i}(t)\right\rangle_{W_{2}^{1}} \\
& =\sum_{i=1}^{n} A_{i}\left\langle\bar{\psi}_{i}(t), T_{j}^{*} \varphi(t)\right\rangle_{W_{2}^{4}}=\sum_{i=1}^{n} A_{i}\left\langle\bar{\psi}_{i}(t), \psi_{j}(t)\right\rangle_{W_{2}^{4}}
\end{aligned}
$$

By orthogonality of $\left\{\bar{\psi}_{i}(t)\right\}_{i=1}^{\infty}$, we have:

$$
\begin{aligned}
& \sum_{l=1}^{j} \beta_{j l} T y_{n}\left(t_{l}\right)=\sum_{i=1}^{n} A_{i}\left\langle\bar{\psi}_{i}(t), \sum_{l=1}^{j} \beta_{j l} \psi_{l}(t)\right\rangle_{W_{2}^{4}} \\
& =\sum_{i=1}^{n} A_{i}\left\langle\bar{\psi}_{i}(t), \bar{\psi}_{j}(t)\right\rangle_{W_{2}^{4}} \\
& A_{j}=\sum_{l=1}^{j} \beta_{j l} F\left(t_{l}, y_{l-1}\left(t_{l}\right), y_{l-1}^{\prime}\left(t_{l}\right), y_{l-1}^{\prime \prime}\left(t_{l}\right)\right)
\end{aligned}
$$

Taking $j=1$, one gets $T y_{n} \quad\left(t_{1}\right)=$ $F\left(t_{1}, y_{0}\left(t_{1}\right), y_{1}^{\prime}\left(t_{1}\right), y_{0}^{\prime \prime}\left(t_{1}\right)\right)$. Taking $j=2$, one gets:

$$
T y_{n}\left(t_{2}\right)=F\left(t_{2}, y_{1}\left(t_{2}\right), y_{1}^{\prime}\left(t_{2}\right), y_{1}^{\prime \prime}\left(t_{2}\right)\right)
$$

Therefore via mathematical induction, we can get that $T y_{n}\left(t_{j}\right)=F\left(t_{j}, y_{j-1}\left(t_{j}\right), y_{j-1}^{\prime}\left(t_{j}\right), y_{j-1}^{\prime \prime}\left(t_{j}\right)\right)$.

\section{Lemma 4}

Let $F\left(t, v_{1}, v_{2} ; v_{3}\right)$ be continuous function in $[0,1]$ with respect to $t, v_{i}, t \in[0,1]$, where $v_{i} \in(-\infty, \infty)$ for $i=$ 1,2 , 3. If $\left\|y_{n}-y\right\|_{W_{2}^{4}} \rightarrow 0, t_{n} \rightarrow t$ as $n \rightarrow 1$, then $F\left(t_{n}, y_{n-1}\left(t_{n}\right), y_{n-1}^{\prime}\left(t_{n}\right), y_{n-1}^{\prime \prime}\left(t_{n}\right)\right) \rightarrow F\left(t, y(t), y^{\prime}(t), y^{\prime \prime}(t)\right)$ as $n \rightarrow 1$.

\section{Proof}

Since $\left\|y_{n}-y\right\|_{W_{2}^{4}} \rightarrow 0$ as $n \rightarrow 1$, by Corollary .1 , it follows that $y_{n-1}^{(i)}(t)$ is converging uniformly to $y^{(i)}(t), i=$ $0,1,2$. Hence, the proof is completed since $\mathrm{F}$ is continuous.

\section{Theorem 4}

Let $\left\{t_{i}\right\}_{i=1}^{\infty}$ be dense on $[0,1]$ and $\left\|y_{n}\right\|_{W_{2}^{3}}$ be bounded, then $y_{n}(t)$ in Equation 12 converges to $y(t)$ of Equation 7 and 8 in the space $W_{2}^{4}[0,1]$ such that $y(t)=\sum_{i=1}^{\infty} A_{i} \bar{\psi}_{i}(t)$, where $A_{i}$ is obtained in Equation 13.

\section{Proof}

Firstly, we will show that $\left\{y_{n}\right\}_{i=1}^{\infty}$ in Equation 12 is increasing by sense of the norm of $W_{2}^{4}[0,1]$. Since $\left\{\bar{\psi}_{i}\right\}_{i=1}^{\infty}$ is complete normal orthogonal basis in $W_{2}^{4}[0,1]$, then $\left\|y_{n}\right\|_{W_{2}^{4}}^{2}=\left\langle y_{n}(t), y_{n}(t)\right\rangle_{W_{2}^{4}}=\left\langle\sum_{i=1}^{n} A_{i} \bar{\psi}_{i}(t), \sum_{i=1}^{n} A_{i} \bar{\psi}_{i}(t)\right\rangle_{W_{2}^{4}}$ $=\sum_{i=1}^{n}\left(A_{i}\right)^{2}$. Therefore, $\left\|y_{n}\right\|_{W_{2}^{4}}^{2}$ is increasing.

Secondly, we will show a convergence of $y_{n}(t)$. By Equation (12), $y_{n+1}(t)=y_{n}(t)+A_{n+1} \bar{\psi}_{n+1}(t)$. Hence, we have $\left\|y_{n+1}\right\|_{W_{2}^{4}}^{2}=\left\|y_{n}\right\|_{W_{2}^{4}}^{2}+\left(A_{n+1}\right)^{2}=\left\|y_{n-1}\right\|_{W_{2}^{4}}^{2}+\left(A_{n}\right)^{2}+\left(A_{n+1}\right)^{2}=\ldots=$ $\left\|y_{0}\right\|_{W_{2}^{4}}^{2}+\sum_{i=1}^{n+1}\left(A_{i}\right)^{2}$. Since, the sequence $\left\|y_{n}\right\|_{W_{2}^{4}}^{2}$ is increasing as well as $\left\|y_{n}\right\|_{W_{2}^{4}}$ is bounded, then $\left\|y_{n}\right\|_{W_{2}^{4}}$ is convergent as $n$ $\rightarrow 1$. That is, $\exists$ a constant $\alpha$ such that $\sum_{i=1}^{\infty}\left(A_{i}\right)^{2}=\alpha$. Thus $A_{i}$ $=\sum_{k=1}^{j} \beta_{i k} F\left(t_{k}, y_{k-1}\left(t_{k}\right), y_{k-1}^{\prime}\left(t_{k}\right), y_{k-1}^{\prime \prime}\left(t_{k}\right)\right) \in l^{2}, i=1,2, \ldots$. On the other hand, since $\left(y_{m}-y_{m-1}\right) \perp\left(y_{m-1}-y_{m-2}\right) \perp \ldots \perp\left(y_{n+1}-y_{n}\right)$ it implies that $\left\|y_{m}-y_{n}\right\|_{W_{2}^{4}}^{2}=$ $\left\|y_{m}-y_{m-1}+y_{m-1}-\ldots+y_{n+1}-y_{n}\right\|_{W_{2}^{4}}^{2}=\left\|y_{m}-y_{m-1}\right\|_{W_{2}^{4}}^{2}+\ldots+$ $\left\|y_{n+1}-y_{n}\right\|_{W_{2}^{4}}^{2}$ for $m>n$. Furthermore, $\left\|y_{m}-y_{m-1}\right\|_{W_{2}^{4}}^{2}=\left(A_{m}\right)^{2}$. Anyhow, $\left\|y_{m}-y_{n}\right\|_{W_{2}^{4}}^{2}=\sum_{i=n+1}^{m}\left(A_{i}\right)^{2} \rightarrow 0$ as $n, m \rightarrow 1$. From completeness of $W_{2}^{4}[0,1]$, for $n \rightarrow 1, \exists y \in W_{2}^{4}[0,1]$ such that $y_{n}(t) \rightarrow y(t)$ in the sense of \|\|$_{W_{2}^{4}}$. Finally, we will show that $y(t)$ is analytic solution of Equation 7 and 8 .

Let $\left\{t_{i}\right\}_{j=1}^{\infty}$ be dense on $[0,1]$, then there is a subsequence $\left\{t_{n_{j}}\right\}_{j=1}^{\infty}$ such that $t_{n_{j}} \rightarrow t$ as $j \rightarrow \infty, \forall t \in[0$, 1]. It is clear, by Lemma .3 , that $T y\left(t_{n_{j}}\right)=$ $F\left(t_{n_{j}}, y_{n_{j}-1}\left(t_{k}\right), y_{n_{j}-1}^{\prime}\left(t_{k}\right), y_{n_{j}-1}^{\prime \prime}\left(t_{k}\right)\right)$. From lemma .4 and continuity of $F$, it follows for $j \rightarrow \infty$ that $T y(t)=F(t$, $\left.y(t) ; y^{\prime}(t), y^{\prime \prime}(t)\right)$. That is, $y(t)$ satisfies Equation 7. As well as if $\bar{\psi}_{i}(t) \in W_{2}^{4}[0,1]$, then $y(t)$ satisfy periodic conditions of Equation 8, which means that $y(t)$ is analytic solution of Equation 7 and 8 such that $y(t)=$ $\sum_{i=1}^{\infty} A_{i} \bar{\psi}_{i}(t)$.

\section{Computational Algorithm and Numerical Experiments}

Using RKHS method, taking $t_{i}=\frac{i-1}{n-1}, i=1,2, \ldots, n$ and according to reproducing kernel functions $K_{s}(t)$ and $Q_{s}(t)$ 
on $[0,1]$; some tabulate results are presented quantitatively at some selected grid points on $[0,1]$ to illustrate the accuracy of the FRK method for handling the PBVP.

\section{Example 1}

Meditate in the following linear differential equation:

$$
y^{\prime \prime \prime}(t)+t y^{\prime \prime}(t) f(t), 0 \leq t \leq 1
$$

with periodic conditions:

$$
y(0)-y(1)=0, y^{\prime}(0)-y^{\prime}(1)=0, y^{\prime \prime}(0)-y^{\prime \prime}(1)=0
$$

where, $f(t)$ is given to obtain the exact solution as $y(t)=e^{t^{2}(1-t)^{2}}$.

\section{Example 2}

Meditate in the following nonlinear diferential equation:

$$
\begin{aligned}
& y^{\prime \prime \prime}(t)-\cos (t)\left(y^{\prime \prime}(t)\right)^{3}+2 \sinh (y(t)) \cosh \left(y(t) y^{\prime}(t)\right) \\
& =f(t), 0 \leq t \leq 1
\end{aligned}
$$

with periodic conditions:

$$
y(0)-y(1)=0, y^{\prime}(0)-y^{\prime}(1)=0, y^{\prime \prime}(0)-y^{\prime \prime}(1)=0
$$

where, $f(t)$ is given to obtain the exact solution as $y(t)=\frac{1}{2} t^{2}(1-t)^{2}$.

\section{Example 3}

Meditate in the following nonlinear differential equation:

$$
y^{\prime \prime \prime}(t)+y^{\prime \prime}(t)+t\left(y^{\prime}(t)\right)^{2}-\cosh ^{-1}(y(t))=f(t), 0 \leq t \leq 1
$$

with periodic conditions:

$$
y(0)-y(1)=0, y^{\prime}(0)-y^{\prime}(1)=0, y^{\prime \prime}(0)-y^{\prime \prime}(1)=0
$$

where, $f(t)$ is given to obtain the exact solution as $y(t)=$ $\cosh \left(t^{2}-t\right)$.

The agreement between the analytical-numerical solutions is investigated for Examples 1, 2 and 3 at various $t$ in $[0,1]$ by computing absolute errors and relative errors of numerically approximating their analytical solutions as shown in Tables 1 to 3, respectively.

Table 1. The analytical-numerical solutions and errors for Example 1

\begin{tabular}{lllll}
\hline$t$ & $y(t)$ & $y_{51}(t)$ & $\left|y(t)-y_{51}(t)\right|$ & $|y(t)|^{-1}\left|y(t)-y_{51}(t)\right|$ \\
\hline 0 & 1.0000000000000000 & 1.0000000000000000 & 0 & 0 \\
0.1 & 1.0081328937531524 & 1.0081340879367722 & $1.19418 \times 10^{-6}$ & $1.18455 \times 10^{-6}$ \\
0.2 & 1.0259304941903822 & 1.0259325793058158 & $2.08512 \times 10^{-6}$ & $2.03241 \times 10^{-6}$ \\
0.3 & 1.0450868583490185 & 1.0450883361164949 & $1.47777 \times 10^{-6}$ & $1.41401 \times 10^{-6}$ \\
0.4 & 1.0592911944779007 & 1.0592914256899810 & $2.31212 \times 10^{-7}$ & $2.18271 \times 10^{-7}$ \\
0.5 & 1.0644944589178593 & 1.0644940798919150 & $3.79026 \times 10^{-7}$ & $3.56062 \times 10^{-7}$ \\
0.6 & 1.0592911944779007 & 1.0592914066684471 & $2.12191 \times 10^{-7}$ & $2.00314 \times 10^{-7}$ \\
0.7 & 1.0450868583490185 & 1.0450883036692906 & $1.44532 \times 10^{-6}$ & $1.38297 \times 10^{-6}$ \\
0.8 & 1.0259304941903820 & 1.0259325450574925 & $2.05087 \times 10^{-6}$ & $1.99903 \times 10^{-6}$ \\
0.9 & 1.0081328937531522 & 1.0081340662518540 & $1.17250 \times 10^{-6}$ & $1.16304 \times 10^{-6}$ \\
1 & 1.0000000000000000 & 1.0000000000000000 & 0 & 0 \\
\hline
\end{tabular}

Table 2. The analytical-numerical solutions and errors for Example 2

\begin{tabular}{lllll}
\hline$t$ & $y(t)$ & $y_{36}(t)$ & $\left|y(t)-y_{36}(t)\right|$ & $|y(t)|^{-1}\left|y(t)-y_{36}(t)\right|$ \\
\hline 0 & 0.00000 & 0.000000000000000000 & 0 & Indeterminate \\
0.1 & 0.00405 & 0.004049976999474401 & $2.30005 \times 10^{-8}$ & $5.67914 \times 10^{-6}$ \\
0.2 & 0.01280 & 0.012799927364010002 & $7.26360 \times 10^{-8}$ & $5.67469 \times 10^{-6}$ \\
0.3 & 0.02205 & 0.022049874903704060 & $1.25096 \times 10^{-7}$ & $5.67330 \times 10^{-6}$ \\
0.4 & 0.02880 & 0.028799836624554605 & $1.63375 \times 10^{-7}$ & $5.67276 \times 10^{-6}$ \\
0.5 & 0.03125 & 0.031249822731293803 & $1.77269 \times 10^{-7}$ & $5.67260 \times 10^{-6}$ \\
0.6 & 0.02880 & 0.028799836624554600 & $1.63375 \times 10^{-7}$ & $5.67276 \times 10^{-6}$ \\
0.7 & 0.02205 & 0.022049874903704060 & $1.25096 \times 10^{-7}$ & $5.67330 \times 10^{-6}$ \\
0.8 & 0.01280 & 0.012799927364010000 & $7.26360 \times 10^{-8}$ & $5.67469 \times 10^{-6}$ \\
0.9 & 0.00405 & 0.004049976999474354 & $2.30005 \times 10^{-8}$ & $5.67914 \times 10^{-6}$ \\
1 & 0.0000 & 0.000000000000000000 & 0 & Indeterminate \\
\hline
\end{tabular}


Table 3. The analytical-numerical solutions and errors for Example 3

\begin{tabular}{lllll}
\hline$t$ & $y(t)$ & $y_{26}(t)$ & $\left|y(t)-y_{26}(t)\right|$ & $|y(t)|^{-1}\left|y(t)-y_{26}(t)\right|$ \\
\hline 0 & 1.0000000000000000 & 1.0000000000000000 & 0 & 0 \\
0.1 & 1.0040527344882193 & 1.0040527445966834 & $1.01085 \times 10^{-8}$ & $1.00677 \times 10^{-8}$ \\
0.2 & 1.0128273299790107 & 1.0128269444788909 & $3.85500 \times 10^{-7}$ & $3.80618 \times 10^{-7}$ \\
0.3 & 1.0221311529634651 & 1.0221299798303292 & $1.17313 \times 10^{-6}$ & $1.14773 \times 10^{-6}$ \\
0.4 & 1.0289385056939790 & 1.0289365747582975 & $1.93094 \times 10^{-6}$ & $1.87663 \times 10^{-6}$ \\
0.5 & 1.0314130998795732 & 1.0314108598211662 & $2.24006 \times 10^{-6}$ & $2.17183 \times 10^{-6}$ \\
0.6 & 1.0289385056939790 & 1.0289365727521844 & $1.93294 \times 10^{-6}$ & $1.87858 \times 10^{-6}$ \\
0.7 & 1.0221311529634651 & 1.0221299763027610 & $1.17666 \times 10^{-6}$ & $1.15118 \times 10^{-6}$ \\
0.8 & 1.0128273299790107 & 1.0128269404245114 & $3.89554 \times 10^{-7}$ & $3.84621 \times 10^{-7}$ \\
0.9 & 1.0040527344882193 & 1.0040527415434230 & $7.05520 \times 10^{-9}$ & $7.02673 \times 10^{-9}$ \\
1 & 1.0000000000000000 & 1.0000000000000000 & 0 & 0 \\
\hline
\end{tabular}

\section{Concluding Summary}

In this article, we introduce the fitting reproducing kernel approach to enlarge its application range for treating a class of third-order periodic BVPs in a favorable reproducing kernel Hilbert space. The method does not require discretization of the variables as well as it provides best solutions in a less number of iterations and reduces the computational work. Further, we can conclude that the presented method is powerful and efficient technique in finding approximate solution for both linear and nonlinear problems. In the proposed algorithm, the solution and its approximation are represented in the form of series in $W_{2}^{4}[0,1]$. The approximate solution and its derivative converge uniformly to exact solution and its derivative, respectively.

\section{Acknowledgment}

The authors express their thanks to unknown referees for the careful reading and helpful comments.

\section{Author's Contributions}

All authors completed the paper together as well as read and approved the final manuscript.

\section{Ethics}

The authors declare that there is no conflict of interests regarding the publication of this paper.

\section{References}

Abu Arqub, O. and M. Al-Smadi, 2014. Numerical algorithm for solving two-point, second-order periodic boundary value problems for mixed integrodifferential equations. Applied Math. Comput., 243: 911-922. DOI: 10.1016/j.amc.2014.06.063

Abu Arqub, O., 2015. Reproducing kernel algorithm for the analytical-numerical solutions of nonlinear systems of singular periodic boundary value problems. Math. Problems Eng., 2015: 518406-518418.

DOI: $10.1155 / 2015 / 518406$
Abu Arqub, O., M. Al-Smadi and N. Shawagfeh, 2013. Solving Fredholm integro-differential equations using reproducing kernel Hilbert space method. Applied Math. Comput., 219: 8938-8948.

DOI: 10.1016/j.amc.2013.03.006

Abu Arqub, O., M. Al-Smadi and S. Momani, 2012. Application of reproducing kernel method for solving nonlinear Fredholm-Volterra integrodifferential equations. Abs. Applied Anal., 2012: 839836-839851. DOI: 10.1155/2012/839836

Abu Arqub, O., M. Al-Smadi, S. Momani and T. Hayat, 2015. Numerical solutions of fuzzy differential equations using reproducing kernel Hilbert space method. Soft Comput.

DOI: $10.1007 / \mathrm{s} 00500-015-1707-4$

Abu-Gdairi, R. and M. Al-Smadi, 2013. An efficient computational method for 4th-order boundary value problems of Fredholm IDEs. Applied Math. Sci., 7: 4761-4774. DOI: 0.12988/ams.2013.37384

Abu-Gdairi, R., M. Al-Smadi and G. Gumah, 2015. An expansion iterative technique for handling fractional differential equations using fractional power series scheme. J. Math. Stat., 11: 29-38.

DOI: $10.3844 /$ jmssp.2015.29.38

Abuteen, E., A. Freihat, M. Al-Smadi, H. Khalil and R.A. Khan, 2016. Approximate series solution of non-linear, fractional klein gordon equations using fractional reduced differential transform method. J. Math. Stat., 12: 23-33.

DOI: $10.3844 / \mathrm{jmssp} .2016 .23 .33$

Ahmad, M., S. Momani, O. Abu Arqub, M. Al-Smadi and A. Alsaedi, 2016. An efficient computational method for handling singular second-order, three points Volterra integro-differenital equations. J. Comput. Theoretical Nanosci.

Al-Smadi, M. and Z. Altawallbeh, 2013. Solution of system of fredholm integro-differential equations by RKHS method. Int. J. Contemporary Math. Sci., 8: 531-540.

Al-Smadi, M., 2013. Solving initial value problems by residual power series method. Theor. Math. Applic., 3: 199-210. 
Al-Smadi, M., A. Freihat, M. Abu Hammad, S. Momani and O. Abu Arqub, 2016. Analytical approximations of partial differential equations of fractional order with multistep approach. J. Comput. Theoretical Nanosci.

Al-Smadi, M., A. Freihat, O. Abu Arqub and N. Shawagfeh, 2015. A novel multi-step generalized differential transform method for solving fractionalorder Lu chaotic and hyperchaotic systems. J. Comput. Anal. Applic., 19: 713-724.

Al-Smadi, M., O. Abu Arqub and A. El-Ajuo, 2014. A numerical iterative method for solving systems of first-order periodic boundary value problems. J. Applied Math., 2014: 135465-135474.

DOI: $10.1155 / 2014 / 135465$

Al-Smadi, M., O. Abu Arqub and S. Momani, 2013. A computational method for two-point boundary value problems of fourth-order mixed integrodifferential equations. Math. Problems Eng., 2013: 832074-832083. DOI: 10.1155/2013/832074

Al-Smadi, M., O. Abu Arqub and N. Shawagfeh, 2012. Approximate solution of BVPs for 4th-order IDEs by using RKHS method. Applied Math. Sci., 6: 2453-2464.

Ashyralyev, A., D. Arjmand and M. Koksal, 2009. Taylor's decomposition on four points for solving third-order linear time-varying systems. J. Franklin Inst., 346: 651-662. DOI: $10.1016 /$ j.jfranklin.2009.02.017

Bushnaq, S., B. Maayah, S. Momani, O. Abu Arqub and M. Al-Smadi et al., 2016. Analytical simulation of singular second-order, three points BVPs for Fredholm operator using computational kernel algorithm. J. Comput. Theor. Nanosci.

Chu, J. and Z. Zhou, 2006. Positive solutions for singular non-linear third-order periodic boundary value problems. Nonlinear Anal., 64: 1528-1542. DOI: $10.1016 /$ j.na.2005.07.005

El-Ajou, A., O. Abu Arqub and M. Al-Smadi, 2015. A general form of the generalized Taylor's formula with some applications. Applied Math. Comput., 256: 851-859. DOI: 10.1016/j.amc.2015.01.034

Geng, F. and M. Cui, 2007. Solving singular nonlinear second-order periodic boundary value problems in the reproducing kernel space. Applied Math. Comput., 192: 389-398.

DOI: 10.1016/j.amc.2007.03.016

Geng, F. and M. Cui, 2012. A reproducing kernel method for solving nonlocal fractional boundary value problems. Applied Math. Lett., 25: 818-823. DOI: 10.1016/j.aml.2011.10.025

Geng, F. and S.P. Qian, 2013. Reproducing kernel method for singularly perturbed turning point problems having twin boundary layers. Applied Math. Lett., 26: 998-1004.

DOI: $10.1016 /$ j.aml.2013.05.006
Geng, F., S.P. Qian and M. Cui, 2013. A brief survey on the reproducing kernel method for integral and differential equations. Commun. Fract. Calculus, 4: 50-63.

Geng, F., S.P. Qian and M. Cui, 2015. Improved reproducing kernel method for singularly perturbed differential-difference equations with boundary layer behavior. Applied Math. Comput., 252: 58-63. DOI: 10.1016/j.amc.2014.11.106

Geng, F., S.P. Qian and S. Li, 2014. A numerical method for singularly perturbed turning point problems with an interior layer. J. Comput. Applied Math., 255: 97-105. DOI: 10.1016/j.cam.2013.04.040

Gregu, M., 1987. Third Order Linear Differential Equations. 1st Edn., D. Reidel Pub. Co., Dordrecht, ISBN-10: 9027721939, pp: 270.

Hopkins, B. and N. Kosmatov, 2007. Third-order boundary value problems with sign-changing solutions. Nonlinear Anal., 67: 126-137. DOI: 10.1016/j.na.2006.05.003

Komashynska, I. and M. Al-Smadi, 2014. Iterative reproducing kernel method for solving second-order integrodifferential equations of fredholm type. J. Applied Math., 2014: 459509-459519. DOI: $10.1155 / 2014 / 459509$

Komashynska, I., M. AL-Smadi, A. Ateiwi and A. Al E'damat, 2016a. An oscillation of the solution for a nonlinear second-order stochastic differential equation. J. Comput. Anal. Applic., 20: 860-868.

Komashynska, I., M. Al-Smadi, A. Ateiwi and S. Al-Obaidy, 2016b. Approximate analytical solution by residual power series method for system of fredholm integral equations. Applied Math. Inform. Sci., 10: 975-985.

Komashynska, I., M. Al-Smadi, O. Abu Arqub and S. Momani, 2016c. An efficient analytical method for solving singular initial value problems of nonlinear systems. Applied Math. Inform. Sci., 10: 647-656. DOI: 10.18576/amis/100224

Kong, L. and Y. Wang, 2001. Positive solution of a singular nonlinear third-order periodic boundary value problem. J. Comput. Applied Math., 132: 247-253. DOI: $10.1016 / \mathrm{S} 0377-0427(00) 00325-3$

Liu, Z., J.S. Ume and S.M. Kang, 2007. Positive solutions of a singular nonlinear third order two-point boundary value problem. J. Math. Anal. Applic., 326: 589-601. DOI: 10.1016/j.jmaa.2006.03.030

Minh., F.M., 1998. Periodic solutions for a third order differential equation under conditions on the potential. Portugaliae Math., 55: 475-484.

Moaddy, K., M. AL-Smadi and I. Hashim, 2015. A novel representation of the exact solution for differential algebraic equations system using residual power-series method. Discrete Dynam. Nature Society, 2015: 205207-205218. DOI: $10.1155 / 2015 / 205207$ 
Momani, S., A. Freihat and M. AL-Smadi, 2014. Analytical study of fractional-order multiple chaotic FitzHugh- Nagumo neurons model using multistep generalized differential transform method. Abs. Applied Anal., 2014: 276279-276287.

DOI: $10.1155 / 2014 / 276279$

$\mathrm{Yu}, \mathrm{H}$. and M. Pei, 2010. Solvability of a nonlinear third-order periodic boundary value problem. Applied Math. Lett., 23: 892896. DOI: $10.1016 /$ j.aml.2010.04.005
Zehour, B., B. Abdelkader and B.S. Mohamed, 2008. Existence result for impulsive third order periodic boundary value problems. Applied Math. Comput., 206: 728-737.

DOI: $10.1016 /$ j.amc.2008.09.030 